\title{
CVD Regimen
}

National Cancer Institute

\section{Source}

National Cancer Institute. CVD Regimen. NCI Thesaurus. Code C63525.

A regimen consisting of cisplatin, vinblastine and dacarbazine used for the treatment of advanced-stage or metastatic malignant melanoma. 